\title{
Rendimiento y composición bromatológica de Setaria sphacelata cv Nandi, en tres edades de rebrote
}

\section{Yield and bromatological composition of Setaria sphacelata cv Nandi, in three regrowth ages}

\author{
Rolando Azania年, ${ }^{\text {, }}$ Luis T. Acosta ${ }^{2, b}$, Anibal R. Rodríguez ${ }^{3, c}$, Gilmar H. López ${ }^{1, d}$ \\ ${ }^{1}$ Laboratorio de Nutrición Animal, Escuela Profesional de Ingeniería Zootecnista, Universidad Nacional Daniel Alcides \\ Carrión, Filial Oxapampa, Cerro de Pasco, Perú. \\ ${ }^{2}$ Escuela Profesional de Ingeniería Ambiental, Universidad Nacional Daniel Alcides Carrión, Filial Oxapampa, Cerro de \\ Pasco, Perú. \\ ${ }^{3}$ Instituto Central de Investigación, Proyecto de Investigación de Vacunos Oxapampa, Universidad Nacional Daniel \\ Alcides Carrión, Oxapampa, Perú. \\ a Ing., $\bigotimes$ rolandfox18@gmail.com, (D) https://orcid.org/0000-0002-5130-4923 \\ b Ing., \luistibhy@hotmail.com, (D) https://orcid.org/0000-0002-1276-1777 \\ cM.Sc., \arodriguezv@undac.edu.pe, (iD https://orcid.org/0000-0003-0248-163X \\ dM.Sc., glopeza@undac.edu.pe, 10 https://orcid.org/0000-0002-4492-5756 \\ * Autor de Correspondencia: Tel. +51963629635 \\ http://dx.doi.org/10.25127/riagrop.20213.702
}

http://revistas.untrm.edu.pe/index.php/RIAGROP revista.riagrop@untrm.edu.pe

Recepción: 02 de abril 2021

Aprobación: 04 de junio 2021

Este trabajo tiene licencia de Creative Commons. Attribution-NonCommercial-ShareAlike $\quad 4.0$ International Public License - CC-BY-NC-SA 4.0

\section{Resumen}

El objetivo del trabajo de investigación fue evaluar el efecto de la edad de rebrote en el rendimiento y composición bromatológica del pasto Setaria sphacelata, en dos condiciones de fertilidad del suelo. El experimento se realizó en un área de $361 \mathrm{~m}^{2}$ de pasto establecido en un suelo de textura franco arenosa, con un $\mathrm{pH}$ fuertemente acido (5.15), y un nivel alto de materia orgánica (7.09\%). Se usó un Diseño Completamente Randomizado con arreglo factorial $3 \times 2$ y tres repeticiones, donde los factores en estudio fueron 3 edades de rebrote y 2 niveles de fertilización. Se obtuvieron 6 tratamientos con tres repeticiones, que recibieron riego por aspersión para mantener una humedad del suelo constante durante el estudio. Las variables evaluadas fueron el rendimiento de materia fresca (MF), materia seca (MS), proteína cruda (PC), fibra cruda (FC) y Cenizas. Los resultados 
evidenciaron que hubo efectos de la edad de rebrote y la fertilización $\mathrm{p} \leq 0.05$, mas no así para la interacción de la edad por fertilización. Se concluye que, a medida que avanza la edad del pasto Setaria sphacelata, el rendimiento de materia fresca y seca tienden a incrementarse, pero su valor nutricional se ve afectado en la disminución de los valores de proteína (PC) y el aumento del valor de fibra (FC). Además, la fertilización produce efectos en la producción de materia fresca y seca que incrementan los rendimientos significativamente, pero reduce el porcentaje de cenizas.

Palabras clave: Análisis bromatológico, fibras, matéria seca, pastos.

\begin{abstract}
The purpose of the research work was to evaluate the effect of regrowth age on the yield and bromatological composition of the Setaria sphacelata grass, in two soil fertility conditions. The experiment was carried out in an area of $361 \mathrm{~m}^{2}$ of pasture established in a sandy loam soil texture, with a strongly acidic $\mathrm{pH}$ (5.15), and a high level of organic matter (7.09\%). Was used a completely Randomized Design with $3 \times 2$ factorial arrangements and 3 repetitions, where the factors under study were 3 regrowth ages and 2 levels of fertilization, thus obtaining 6 treatments with three replications, which received sprinkler irrigation to maintain constant soil moisture during the study. The variables evaluated were the yield of fresh matter (MF), dry matter (DM), raw protein (PC), raw fiber (FC), and Ash. The results showed that there were effects of regrowth age and fertilization $\mathrm{p} \leq 0.05$, but not for the interaction of age by fertilization. It is concluded that as the age of the Setaria sphacelata grass progress, the yield of fresh and dry matter tends to increase, but its nutritional value is affected by decreasing the protein (PC) values and increasing the fiber of value (FC); the Fertilization produces to defects on the production of fresh and dry matter, significantly increasing yields, but reduces the percentage of ash.
\end{abstract}

Keywords: Bromatological analysis, fibers, dry matter, grasses.

\section{INTRODUCCIÓN}

Los pastos constituyen la principal fuente de nutrimentos para la alimentación del ganado bovino en las regiones tropicales. Indudablemente, el principal atributo de los pastos tropicales es su gran capacidad para producir materia seca y, por ende, son ideales para suministrar proteína, energía, minerales, vitaminas y fibra al ganado bovino especializado en la producción de leche, así como al de doble propósito y de carne (Sánchez, 2007). Gran parte de las áreas de la provincia de Oxapampa son destinadas a pasturas establecidas. Actualmente, la superficie de pasto setaria (Setaria sphacelata) instalada fortuitamente en el distrito de Oxapampa es de 5650 hectáreas, el distrito de Chontabamba con 2960 hectáreas y Huancabamba con 6650 hectáreas (MINAGRI, 2015).

El pasto setaria es una de las especies que más se ha adaptado a las diferentes condiciones edafoclimáticas de esta realidad se caracteriza por adaptarse en zonas que reciben más de 600 $\mathrm{mm}$ de lluvia anualmente. Además, resiste la sequía y el encharcamiento y prefiere suelos húmedos y fértiles, pero se desarrolla bien en suelos pobres (Bernal, 1994). Uno de los factores que incide en la calidad de los pastos es la edad 
porque a medida que aumenta se presenta incrementos en la producción de materia seca acompañados por componentes de la pared celular (fibra y lignina) y disminución de proteínas y carbohidratos no estructurales. Por ello, urge hacer estudios de la composición química de los pastos para recomendar una edad óptima para el mejor aprovechamiento de los pastos (Sánchez, 2007). La respuesta de los pastos a la fertilización se expresa de diferente manera, el efecto más notable de la fertilización es el incremento en el rendimiento de materia seca. La aplicación de nutrientes afecta también la calidad del forraje que se mide evaluando diferentes parámetros como el contenido de proteína, minerales o por las variaciones en la digestibilidad del pasto (Bernal y Espinoza, 2003).

El objetivo del presente trabajo fue evaluar el efecto de la edad de rebrote en el rendimiento y composición bromatológica del pasto setaria (Setaria sphacelata) con diferentes condiciones de fertilización. Para poder cumplir con el objetivo general, se plantearon los objetivos específicos: Evaluar el efecto de la edad de rebrote en el rendimiento de materia fresca y seca del pasto setaria (Setaria sphacelata) con diferentes condiciones de fertilización. Evaluar el efecto de la edad de rebrote en el contenido de proteína cruda y fibra bruta del pasto setaria (Setaria sphacelata) con diferentes condiciones de fertilización. Evaluar el efecto de la edad de rebrote en el contenido de cenizas del pasto setaria (Setaria sphacelata) con diferentes condiciones de fertilización.

\section{MATERIALES Y MÉTODOS}

\subsection{Lugar de estudio}

El trabajo de investigación se realizó en el Fundo: Sociedad Ganadera "EL SEQUIÓN", que se encuentra ubicado en el sector Gramazú, en el distrito de Huancabamba, provincia de Oxapampa, departamento de Pasco y se realizó entre el mes de junio y octubre 2016.

\subsection{Características del suelo}

El suelo donde se desarrolló el experimento es considerado de clase textural franco arenoso (Fr.A.), la reacción del suelo es fuertemente ácido ( $\mathrm{pH}=5.15)$, característicos de los suelos del trópico. El porcentaje de materia orgánica se encuentra alto $(\mathrm{MO}=7.09)$, mientras que el fósforo se encuentra de bajo a medio, al igual que el potasio se encuentra en niveles bajos, el CIC se encuentra por debajo de los rangos ideales, la suma de bases es inferior a $5 \mathrm{cmol}$ $(+) / \mathrm{kg}$ por lo que se le puede relacionar con suelos de baja fertilidad.

Se utilizó el valor de densidad aparente de 1.6 $\mathrm{g} / \mathrm{cm}^{3}$, una profundidad de suelo de $0.15 \mathrm{~cm}$ y la tasa de mineralización de $2 \%$ para climas medios estimadas por Minag (2011) y con los resultados de la tabla 1 , se realizó los cálculos correspondientes de nutrientes en $\mathrm{Kg} /$ ha según la metodología descritas por Cuesta y Villanedas (2002) y se hallaron valores de $\mathrm{N}$ asimilable de $170.16 \mathrm{Kg} / \mathrm{ha}, 41.17 \mathrm{Kg}$ de P2O5/ha y 237.14 Kg de K2O/ha. 
Tabla 1. Análisis de suelos: Caracterización

\begin{tabular}{|c|c|c|c|c|c|c|c|c|}
\hline $\mathrm{pH}$ & CE dS.m ${ }^{-1}$ & $\mathrm{CaCO} 3 \%$ & $\mathrm{MO} \%$ & P ppm & K ppm & Arena \% & Limo \% & Arcilla \% \\
\hline 5.15 & 0.13 & 0 & 7.09 & 7.6 & 82 & 67 & 24 & 9 \\
\hline CIC & $\mathrm{Ca}^{2+}$ & $\mathrm{Mg}^{2+}$ & $\mathrm{K}^{+}$ & $\mathrm{Na}^{+}$ & $\mathrm{AL}^{+}+\mathrm{H}^{+}$ & $\begin{array}{l}\text { Suma de } \\
\text { Cationes } \\
\end{array}$ & $\begin{array}{c}\text { Suma de } \\
\text { Bases } \\
\end{array}$ & $\begin{array}{l}\text { \% Sat. } \\
\text { Bases }\end{array}$ \\
\hline \multicolumn{9}{|c|}{$\mathrm{meq} / 100 \mathrm{~g}$} \\
\hline 19.68 & 2.6 & 0.78 & 0.24 & 0.17 & 0.5 & 4.28 & 3.78 & 19 \\
\hline
\end{tabular}

Fuente: (LASPAF, 2016)

\subsection{Tratamientos}

Se estudiaron 6 tratamientos resultantes de la combinación de 3 periodos de rebrote $\left(R_{1}=30\right.$ días de rebrote, $R_{2}=45$ días de rebrote, $R_{3}=60$ días de rebrote) y 2 niveles de fertilización ( $\mathrm{F} 0=$ sin fertilización, F1= con fertilización). Se utilizó el Diseño Completo Randomizado con arreglo factorial $3 \times 2$ con 3 repeticiones.

\subsection{Parámetros evaluados}

2.4.1. Rendimiento de materia fresca (MF) $\mathrm{kg} / \mathrm{ha}: \mathrm{El}$ follaje se recolectó a una altura de $10 \mathrm{~cm}$ del nivel del suelo, dentro del marco de un metro cuadrado, se midió dicho peso y se estimó a una hectárea.

2.4.2. Rendimiento de materia seca (MS) $\mathrm{kg} / \mathrm{ha}$ : Se pesaron 200 gramos de muestra y se secó en una estufa por 48 horas a $60^{\circ} \mathrm{C}$, hasta obtener un peso constante $\mathrm{y}$ por diferencia de peso se determinó el porcentaje de materia seca.

2.4.3. Proteína y Fibra cruda (\%): Las muestras fueron analizadas en el Laboratorio de Evaluación Nutricional de Alimentos (LENA) de la Universidad Nacional Agraria La Molina y la medición de Cenizas (\%) se realizó mediante el método AOAC 942.05 (AOAC, 2005).

\subsection{Análisis estadístico}

Se realizó el análisis mediante el Software SAS, sobre el análisis de varianza con un nivel de significancia de $\mathrm{p}<0.05$ y las comparaciones múltiples entre de medias encontradas mediante la prueba de Duncan $(\mathrm{p}<0.05)$.

\section{RESULTADOS Y DISCUSIÓN}

\subsection{Rendimiento de materia fresca (MF)}

En la tabla 2, se muestra los resultados del rendimiento de $\mathrm{MF}$ en $\mathrm{kg} / \mathrm{h}$, donde se encontró diferencias significativas entre las medias de producción de materia fresca, que alcanzó el mayor rendimiento a los 60 días en pastos fertilizados $\mathrm{y}$ sin fertilización tal como se muestra en la tabla 3, pero, a los días 30 y 45 no existen diferencias. Estos resultados manifiestan claramente el gran efecto que produce la edad de rebrote en la producción de biomasa ya sea en diferentes condiciones de fertilidad de suelo.

Situaciones similares fueron reportados por Sánchez (2011), quien al evaluar la biomasa fresca del pasto setaria a los 120, 135, y 150 días después de la siembra, obtuvo producciones de $10.084,18.878$ y 23.010 Ton/ha de biomasa fresca 
afirmando que la producción de biomasa es progresiva a las edades de corte.

Los pastos al igual que todas las plantas requieren de macro y micronutrientes para formar sus estructuras. Si estos nutrientes no se encuentran disponibles en el suelo, el crecimiento y el desarrollo del pasto se ve afectado. La influencia de la fertilización causa efectos en la producción porque logra siempre incrementar la producción de biomasa de las pasturas. Estos resultados son confirmados por Egg (1990) quien reporta producciones de 4.8 y 7.37 TM/ha para setaria sin fertilización $y$ fertilizado cortado a los 45 días de rebrote, datos superiores a los encontrados en esta investigación.

Tabla 2. Parámetros físico-químicos del pasto setaria a diferentes días de rebrote

\begin{tabular}{lrrrrrr}
\hline \multirow{2}{*}{ Parámetros } & \multicolumn{2}{c}{ 30 DÍAS } & \multicolumn{2}{c}{ 45 DÍAS } & \multicolumn{2}{c}{ 60 DÍAS } \\
\cline { 2 - 7 } & $\begin{array}{c}\text { Sin } \\
\text { fertilización }\end{array}$ & $\begin{array}{c}\text { Con } \\
\text { fertilización }\end{array}$ & $\begin{array}{c}\text { Sin } \\
\text { fertilización }\end{array}$ & $\begin{array}{c}\text { Con } \\
\text { fertilización }\end{array}$ & $\begin{array}{c}\text { Sin } \\
\text { fertilización }\end{array}$ & $\begin{array}{c}\text { Con } \\
\text { fertilización }\end{array}$ \\
\hline $\mathrm{MF} \mathrm{Kg} / \mathrm{Ha}$ & $1973.7 \mathrm{~b}$ & $4792.0 \mathrm{~b}$ & $3084.7 \mathrm{ab}$ & $5354.2 \mathrm{~b}$ & $3985.7 \mathrm{a}$ & $7987.1 \mathrm{a}$ \\
$\mathrm{MS} \mathrm{Kg} / \mathrm{Ha}$ & $469.8 \mathrm{~b}$ & $996.9 \mathrm{~b}$ & $702.4 \mathrm{a}$ & $1097.6 \mathrm{~b}$ & $905.8 \mathrm{a}$ & $1639.4 \mathrm{a}$ \\
$\mathrm{PC} \%$ & $12.2 \mathrm{a}$ & $14.8 \mathrm{a}$ & $10.4 \mathrm{ab}$ & $10.5 \mathrm{~b}$ & $9.8 \mathrm{~b}$ & $10.1 \mathrm{~b}$ \\
$\mathrm{FC} \%$ & $21.2 \mathrm{~b}$ & $21.5 \mathrm{~b}$ & $23.3 \mathrm{a}$ & $23.7 \mathrm{ab}$ & $24.9 \mathrm{a}$ & $25.3 \mathrm{a}$ \\
CENIZA \% & $12.5 \mathrm{a}$ & $10.9 \mathrm{a}$ & $12.7 \mathrm{a}$ & $10.0 \mathrm{a}$ & $12.5 \mathrm{a}$ & $10.8 \mathrm{a}$ \\
\hline
\end{tabular}

Promedios en la misma columna con letras $(\mathrm{a}, \mathrm{b}, \mathrm{c})$ distintas difieren entre sí $(\mathrm{P}<0.05)$

\subsection{Rendimiento de materia seca (MS)}

Se observó que los factores edad de rebrote y fertilización alcanzaron significación estadística $(\mathrm{p} \leq 0.05)$. Además, el rendimiento de materia seca aumentó a medida que avanzó la edad. El pasto setaria ( $S$. sphacelata) fertilizado no muestran diferencias significativas entre las edades de rebrote de 30 y 45 días, pero sí a los 60 días. Adicionalmente, el pasto setaria sin fertilizar no muestra diferencias significativas entre los días 45 y 60 pero sí a los 30 días de rebrote, tal como se detalla en la tabla 2. sin embargo, la interacción del factor edad de rebrote por fertilización no demostró diferencias estadísticas significativas (veáse tabla 3).

El incremento de MS con la edad de rebrote está relacionado con el aumento del proceso fotosintético que ocurre en la planta y con ello la síntesis de carbohidratos estructurales, lo que incrementa la MS de los pastos, tal como menciona Miller (1989), quien afirma que una elevada proporción del crecimiento inicial está constituido por hojas, y a medida que las plantas maduran las paredes de los tallos se hacen más gruesas y se lignifican.

Asimismo, Bernal (1994) manifiesta que a medida que aumenta la edad del pasto se presenta grandes aumentos en la producción de materia seca acompañados por incrementos en componentes de la pared celular (fibra y lignina).

Los resultados del incremento de la materia seca con la edad de rebrote coinciden con los encontrados por Aguilar (1996), quien reporta rendimientos para el pasto setaria de 1589.2, 1745.2, 1935.9 Kg/MS/ha, a los 28, 35 y 42 días de 
rebrote y afirman que al aumentar los días de corte se incrementa la producción de forraje.

Similares resultados fueron obtenidos por Verdecía et al. (2008), Ramírez et al. (2010) y Bendersky et al. (2006), en experimentos en diferentes pastos tropicales, que afirman que la edad de rebrote tiene efectos en la producción de materia seca que se incrementan a medida que esta avanza.
Bernal y Espinoza (2003) mencionan que un suelo fértil y productivo debe contener todos los elementos minerales esenciales para las plantas en cantidades suficientes y en proporciones balanceadas. Cuando no se cumple alguna de las condiciones anteriores, el crecimiento del forraje se inhibe y la especie no puede mostrar todo su potencial.

Tabla 3. Parámetros físico-químicos del pasto setaria a diferentes condiciones de fertilización

\begin{tabular}{|c|c|c|c|c|c|c|}
\hline \multirow{2}{*}{ Parámetros } & \multicolumn{3}{|c|}{ Sin fertilización } & \multicolumn{3}{|c|}{ Con fertilización } \\
\hline & 30 & 45 & 60 & 30 & 45 & 60 \\
\hline $\mathrm{MF} \mathrm{Kg/Ha}$ & $1873.7 b$ & $3084.7 b$ & $3985.7 b$ & $4792.0 \mathrm{a}$ & $5354.2 \mathrm{a}$ & $7987.1 \mathrm{a}$ \\
\hline $\mathrm{MS} \mathrm{Kg} / \mathrm{Ha}$ & $469.8 b$ & $702.4 b$ & $905.8 \mathrm{~b}$ & $996.9 \mathrm{a}$ & $1097.6 \mathrm{a}$ & $1639.4 \mathrm{a}$ \\
\hline $\mathrm{PC} \%$ & $12.2 \mathrm{a}$ & $10.4 \mathrm{a}$ & $9.8 \mathrm{a}$ & $14.8 \mathrm{a}$ & $10.5 \mathrm{a}$ & $10.1 \mathrm{a}$ \\
\hline FC $\%$ & $21.2 \mathrm{a}$ & $23.3 \mathrm{a}$ & $24.9 \mathrm{a}$ & $21.5 \mathrm{a}$ & $23.7 \mathrm{a}$ & $25.3 \mathrm{a}$ \\
\hline CENIZA \% & $12.5 \mathrm{a}$ & $12.7 \mathrm{a}$ & $12.5 \mathrm{a}$ & $10.9 \mathrm{a}$ & $10.0 \mathrm{~b}$ & $10.8 \mathrm{a}$ \\
\hline
\end{tabular}

Promedios en la misma fila con letras $(a, b)$ distintas difieren entre sí $(\mathrm{P}<0.05)$

Los pastos fertilizados muestran un mayor rendimiento de materia seca por hectárea, que confirman el efecto de la fertilización en la producción. Estos resultados son confirmados por Egg (1990) al probar diferentes dosis de fertilizantes de N, P, K, al evaluar a los 45 días en condiciones parecidas a las nuestras obtuvo rendimientos de $1.28 \mathrm{TM} /$ ha de MS para pasto setaria (S. sphacelata) fertilizado y $0.93 \mathrm{TM} /$ ha de MS para el testigo. También, diversos estudios realizados por Mas (2007), Heyn y Valinotty (1996) e Hidalgo (2004), en pastos tropicales, demuestran el efecto positivo de la fertilización en el incremento de materia seca.

\subsection{Proteína cruda (PC)}

La edad de rebrote influyó en los contenidos de proteína del pasto setaria (S. sphacelata). Se encontró diferencias significativas entre los $30 \mathrm{y}$ 60 días de rebrote, tanto para los pastos fertilizados y sin fertilizar. Sin embargo, en los pastos fertilizados se encuentran diferencias entre los días 30 y 45, mas no fue así para los pastos sin fertilización (véase tabla 2).

Los resultados obtenidos concuerdan con lo estudiado por Aguilar (1996), quien, al evaluar el pasto setaria (Setaria splendida) a los $28,35 \mathrm{y}$ 42 días de rebrote, encontró diferencias significativas $(p \leq 0.05)$ en el contenido de proteína cruda 9.02, 8.67 y $8.23 \%$, respectivamente.

Asimismo, Rincón et al. (2008) reporta resultados similares al evaluar el pasto Brachiaria decumbes y Brachiaria brizantha con defoliaciones y se encontraron diferencias significativas $p \leq 0.05$ en los valores de proteína cruda de 12.9, 11.2 y $9.8 \%$ evaluados a los 14,28 
y 42 días de rebrote que afirman que la edad influye en el valor proteico de estos pastos.

Los resultados obtenidos se encuentran por encima de los niveles críticos recomendados por Pírela (2005), quien afirma que cuando los forrajes tropicales presentan porcentajes de proteína cruda menores al $7 \%$ se limita el consumo del animal.

El mayor contenido de proteína en los estadios tempranos de los forrajes pudo deberse a una mayor proporción de hojas, que cumplen una función de síntesis y asimilación de carbohidratos y presentan alta proporción de tejido parenquimatoso localizado en el mesófilo. Esto le imprime características de altos contenidos de nitrógeno y carbohidratos no estructurales y, por consiguiente, elevado valor nutritivo (Trujillo y Uriarte, 2015).

La fertilización no causó efectos significativos en el valor de proteína cruda en este experimento. Estos resultados son similares a los reportados por Ortega y Gonzales (1985) al evaluar diferentes dosis de $\mathrm{N}(0,100,200$ y 300 $\mathrm{kg} / \mathrm{N} / \mathrm{ha}$ ) en pasto estrella (Cynodon nlemfuensis), no hallaron diferencias significativas $\mathrm{p} \leq 0.05$ para los contenidos de proteína cruda, lo que confirma que la fertilización no tuvo efectos sobre el contenido de PC.

\subsection{Fibra cruda (FC)}

Se encontró diferencias estadística $\mathrm{p} \leq 0.05$ para el factor edad de rebrote (tabla 2), mas no se observa significación estadística para el factor fertilización tabla 3 y se observa que la FC aumenta a medida que avanza la edad en las dos condiciones de fertilización.
Del Pozo (2004) afirma que el aumento de la edad de rebrote provoca cambios significativos en los componentes solubles, estructurales y la digestibilidad de los pastos. Todo ello hace que su valor nutritivo disminuya con el avance de la edad, cuya tasa de reducción es mayor en las gramíneas que en las leguminosas.

Van Soest (1994 c.p. Trujillo y Uriarte, 2015) señala que en el trópico donde no hay heladas, hay menos necesidad de reservas y más problemas por depredadores, plagas y enfermedades. Por lo tanto, las plantas tropicales, para poder sobrevivir, invierten sus reservas en estructuras de resistencia.

Estas afirmaciones son respaldadas por Sánchez (2011) en su experimento con pasto setaria, donde obtuvo valores de 35.2, 31.7 y $35.87 \%$ de fibra cruda evaluado a los 120, 135 y 150 días después de la siembra, que confirma que el incremento de fibra es progresivo a las edades de corte.

Estudios realizados en ecuador por Molina (2015) reportan valores de fibra cruda 29.8, 32.87 y $38.38 \%$ para pasto miel (Setaria splendida) fertilizado, si se considera que, a mayor tiempo de corte o pastoreo, el pasto miel tiende a disminuir su valor alimentico.

Ramírez y Pérez (2006) quienes afirman que los contenidos de fibra cruda en los pastos se incrementan con la edad de corte, encontrando diferencia de $35.6 \%$ y $41.5 \%$ de FC al evaluar el pasto Maralfalfa (Pennisetum sp.) sometido a dos fechas de corte 45 y 60 días. Los estudios realizados en pasto setaria muestran valores muy superiores a los obtenidos en el presente estudio los cuales pueden ser debido a la especie y a las condiciones de ese país. 


\subsection{Cenizas (\%)}

Se observó diferencias significativas a los 45 días de rebrote, entre los porcentajes de cenizas del pasto fertilizado (10 \%) y el pasto sin fertilización $(12.7 \%)$, pero no se encontró diferencias significativas en los cortes realizados a los 30 y 60 días de rebrote detallado en la tabla 2.

Los valores de cenizas obtenidos en este experimento son ligeramente inferiores a los reportados por Molina (2015), quien reporta valores $13.43,11.47$ y $11.4 \%$ de cenizas para setaria (Setaria splendida) sin fertilizar, pero en setaria fertilizadas obtuvo 13.13, 11.53 y $12.57 \%$ de cenizas a los 21, 28 y 35 días de rebrote.

Avellaneda et al. (2008), al estudiar tres variedades del pasto Brachiaria (B. decumbens, B. brizantha y B. ruzizienzis 44-6 $\mathrm{x}$ brizantha $\mathrm{cv}$. Marandú), reporta valores promedios de cenizas de 10.51, 10.46 y $11.42 \%$, respectivamente. Al evaluar el efecto de las edades de corte a los 28, 56, 84 y 112 días, se obtuvo valores de cenizas de 12.75, 11.76, 9.45 y $9.22 \%$, no encontró diferencias significativas $(p \leq 0.05)$ entre los cortes realizados a los 28 y 56 días, pero si menciona una disminución del porcentaje de cenizas al transcurrir el tiempo.

Al realizar el corte a los 45 días de rebrote, se obtuvo diferencias significativas entre el pasto fertilizado y sin fertilizar.

Resultados similares fueron reportados por Soto et al. (2005), en su investigación realizada en pasto Kikuyo (Pennisetum clandestinum), quienes afirman contenido de cenizas fue menor en el pasto fertilizado (9.02 \%, p<0.05) comparado con el pasto sin fertilizar (10.03\%).

\section{CONCLUSIONES}

La fertilización y la edad de rebrote incrementaron los rendimientos de materia fresca y seca del pasto setaria y a medida que la edad avanza, la producción tiende a incrementarse.

El porcentaje de proteína cruda se ve afectado por la edad de rebrote, disminuyendo a medida que la edad avanza. La concentración de fibra cruda aumenta su valor a medida que se alarga los días de rebrote. Además, la edad de rebrote no tiene efecto en el porcentaje de ceniza del pasto setaria.

\section{Referencias}

Aguilar D. (1996). Respuesta del pasto setaria (Setaria splendida) a tres niveles de fertilizacion con nitrogeno, tres con magnesio y tres edades de corte. Trabajo de grado academico de licenciatura, Escuela Agricola Panamerlcana departamento de Zootecnia, Honduras.

Avellaneda J., Cabezas F., Quintana R., Luna R., Montañez O., Espinoza I., Pinargote E. (2008). Comportamiento agronómico y composición química de tres variedades de Brachiaria en diferentes edades de cosecha. Ciencia y Tecnologia, 1(2), 87-94.

Bendersky D., Brizuela M., Cid S. y Altuve. (2006). Producción y calidad de forraje diferido de Setaria sphacelata cv Narok fertilizada. INTA EEA Mercedes.

Bernal J. (1994). Pastos y forrajes tropicales ( $3^{\text {ra }}$ edicion ed.). Colombia: Banco ganadero.

Bernal J., y Espinoza J. (2003). Manual de nutrición y fertilización de pastos. Quito, Ecuador: International Plant Nutrition Institute (IPNI).

Cuestas P. y Villaneda E. (2002). El análisis de suelos: toma de muestras y recomendaciones de fertilización para la producción ganadera. Recuperado el 10 de diciembre de 2020, de https://es.scribd.com/doc/92619818/Analisis-deSuelos-Interpretacion

Del Pozo, P. (2004). Bases ecofisiologicas para el manejo de los pastos tropicales. Recuperado el 12 de Febrero de 2019, http://www.produccionanimal.com.ar/produccion_ y_manejo_pasturas/pastoreo\%20sistemas/30bases_e cofisiologicas_manejo_pasturas_tropicales.pdf 
Egg L. (1990). Respuesta a 4 niveles de fertilización, en una pastura establecida de Setarea sphacelata. Tesis de grado, Universidad Nacional Daniel Alcides Carrion, Oxapampa, Perú.

Heyn R. y Valinotty, P. (1996). Efecto de la fertilización nitrogenada y fosfatada sobre la productividad del pasto setaria (Setaria anceps S.) cv. Kazungula. Investigaclón Agraria,1(1), 35 - 40.

Hidalgo J. (2004). Producción de materia seca y contenido de proteína cruda y fibra neutro detergente del pasto Brachiaria híbrido Mulato, en El Zamorano. Proyecto Especial del Programa de Ingeniería en Ciencia y Producción Agropecuaria, Zamorano, Honduras.

Mas. (2007). Programa Nacional Pasturas y Forrajes. Revista INIA, Bogotá - Colombia. Editorial ABC, Pág. $5-6$.

Miller J. (1989). Nutricion y alimentacion del ganado vacuno lechero. Zaragoza, España: Acribia, S.A.

Minag. (2011). Manejo y fertilidad de suelos. Recuperado el 01 de marzo de 2021. http://minagri.gob.pe/portal/download/pdf/herrami entas/cendoc/ manualesboletines/papa/manejo_fertil_suelos_feb11.pdf

MINAGRI. (2015). Datos Estadísticos Agropecuarios de la Provincia de Oxapampa.

Molina J. (2015). Evaluación nutricional del Pasto Miel (Setaria splendida) a los 21, 28 y 35 días con y sin fertilización nitrogenada en Nanegalito, provincia de Pichincha. Trabajo de Grado, Universidad Central del Ecuador, Quito, Ecuador.

Pirela M. (2005). Valor nutritivo de los pastos tropicales. Recuperado el 24 de septiembre de 2020, http://www.avpa.ula ve/docuPDFs/libros_online/manualganaderia/seccion3/articulo6-s3.pdf

Ramirez Y. y Perez J. (2006). Efecto de la edad de corte sobre el rendimiento y composicion quimica del pasto Maralfalfa (Pensisetum sp.). Rev. Unell. Cienc. Tec.24,57-62.

Ramírez., Herrera S., Leonard I., Verdecia D. y Álvarez Y. (2010). Rendimiento de materia seca y calidad nutritiva del pasto Brachiaria brizantha x Brachairia ruziziensis vc. Mulato en el Valle del Cauto, Cuba, Revista Cubana de Ciencia Agrícola,44(1), 65-72.

Rincon A., Ligarreto G. y Garay, E. (2008). Producción de forraje en los pastos Brachiaria decumbens cv. amargo y Brachiaria brizantha cv. toledo, sometidos a tres frecuencias y a dos intensidades de defoliación en condiciones del piedemonte llanero Colombiano, Rev.Fac.Nal.Agr.Medellín 61(1), 4336-4346.

Sanchez J. (2007). Utilización eficiente de las pasturas tropicales en la alimentacion del ganado lechero. http://nutriciondebovinos.com.ar/MD_upload/nutri ciondebovinos_com_ar/Archivos/File/UTILIZACIO N_DE_PASTURAS_TROPICALES_POR_EL_GANA DO_LECHERO.pdf

Sánchez J. (2011). Establecimiento de una pradera de setaria splendida (Setaria sphacelata) para corte, en la finca Punzara de la Universidad Nacional de Loja. Tesis de grado no publicada, Universidad Nacional de Loja, Loja, Ecuador.

Soto C., Valencia A., Galvis R. y Correa. (2005). Efecto de la edad de corte y del nivel de fertilización nitrogenada sobre el valor energético y proteico del pasto kikuyo (Pennisetum clandestinum). Revista Colombina de Ciencias Pecuarias18 (1),17-26.

Trujillo A., y Uriarte G. (2015). Valor nutritivo de las pasturas. Recuperado el 23 de octubre de 2019, http://www.inia.uy/Publicaciones/Documentos\%20c ompartidos/fpta\%2048_2013.pdf

Verdecía D. M., Ramírez J., Leonard I., Pascual Y., López Y. (2008). Rendimiento y componentes del valor nutritivo del Panicum máximum cv. Tanzania, Revista electrónica de Veterinaria,IX(5). 